\title{
EVOLUTION OF SCALAR PERTURBATIONS IN COSMOLOGY WITH QUINTESSENTIAL DARK ENERGY
}

\author{
B. Novosyadlyj, O. Sergijenko \\ Astronomical Observatory of Ivan Franko National University of Lviv, \\ 8, Kyryla i Mefodija St., Lviv, UA-79005, Ukraine \\ novos@astro.franko.lviv.ua,olka@astro.franko.lviv.ua
}

(Received January 30, 2009)

\begin{abstract}
The dynamics of the expansion of the Universe and evolution of scalar perturbations are discussed for the quintessential scalar fields $Q$ with the classical Lagrangian $L=\frac{1}{2} Q_{; i} Q^{; i}-U(Q)$ satisfying the additional condition $w=$ const or $c_{a}^{2}=0$. Both quintessential fields are studied for the same cosmological model. It is shown that the accelerated expansion of the Universe is caused by the effect of the rolling down of the field to the minimum. At the early epoch the contribution to dynamics of the quintessence with $w=$ const is negligible (like that of the cosmological constant) while the quintessence with $c_{a}^{2}=0$ mimics dust matter. In the future a scalar field with $c_{a}^{2}=0$ will mimic the cosmological constant.

The systems of evolution equations for gauge-invariant perturbations of metric, matter and quintessence have been analysed analytically for the early stage of the Universe life and numerically up to the present epoch. It is shown that amplitudes of the adiabatic matter density perturbations grow similarly in both models (likewise in the $\Lambda$ CDM-model), but time dependences of different amplitudes of quintessence perturbations are varied: gauge-invariant variables $D_{g}^{(Q)}$ and $D_{s}^{(Q)}$ decay from the initial constant value after the particle horizon entry while $D^{(Q)}$ and $V^{(Q)}$ grow at an early stage before the horizon entry and decay after that in the quintessence-dominated epoch when the gravitational potential starts to decay so that at the current epoch they are approximately two orders lower than the matter ones on supercluster scales. Therefore, on the subhorizon scales quintessential scalar fields are smoothed out while the matter clusters.

It is also shown that both quintessential scalar fields suppress the growth of matter density perturbations and the amplitude of gravitational potential. In these QCDM-models - unlike $\Lambda$ CDM ones - such a suppression is scale dependent and more visible for the quintessence with $c_{a}^{2}=0$.

Key words: cosmology: theory - dark energy - scalar field - dynamics of expansion of the Universe - evolution of scalar perturbations.
\end{abstract}

PACS number(s): 95.36.+x, 98.80.-k

\section{INTRODUCTION}

Cosmological observations of the last decade suggest that the main part of the energy density of the Universe - more than $70 \%$ - belongs to the unknown essence, called "dark energy". Its cosmological mission is to provide the accelerated expansion of the Universe, revealed from exploration of SN Ia's in distant galaxies and temperature fluctuation power spectrum of cosmic microwave background. The cosmological $\Lambda$ CDMmodel, based on the Einstein equations with the cosmological constant (see $[1,24,43]$ and references therein), describes very well almost the whole set of the observational data on the dynamics of expansion of the Universe and formation of its large-scale structure. But physical interpretation of the cosmological constant is rather problematic $[7,8,34,35,37,42]$. Therefore alternative approaches - new physical fields (classical scalar field - quintessence, tachyon field, k-essence, phantom field, quintom field), Chaplygin gas, gravity and general relativity modifications, multidimensional gravity, branes and others - are being thoroughly studied (see reviews $[6,8,11,19,33,35,37]$ as well as special issue of Gen. Relativ. Gravit., 2008, v. 40). Up till now none of them has a crucial preferability from observational or theoretical point of view. Therefore each of them must be comprehensively studied. Here we restrict ourselves to quintessential scalar fields with classical Lagrangian $L=Q_{; i} Q^{; i} / 2-U(Q)$ in the dark energy - matter dominated Universe.

The quintessence model can be defined by setting the appropriate potential $U(Q)$ or equation of state (EoS) parameter $w_{Q} \equiv p_{Q} / c^{2} \rho_{Q}$. There is a dozen or more physically-motivated shapes of the potential $U(Q)$ : exponential, double exponential, exponential with inverse power, power-law, etc. The dynamics of such scalar fields has been discussed (see review [11]). The EoS parameter of dark energy completely defines the background dynamics as well as the evolution of cosmological perturbations $[21,22,28]$. Since observational data on SN Ia magnitude - redshift relation and cosmic microwave anisotropy give relatively narrow ranges of dark energy density and EoS parameter values, it looks quite attractive to establish the potential $U(Q)$ using these data and analyse the background dynamics and perturbative properties of such a scalar field which have not been studied sufficiently.

In our previous papers we constructed the potentials of scalar fields with classical and tachyonic Lagrangian leading to the constant EoS parameter $w_{Q}=$ const [40] and analysed the background dynamics and perturbative properties of such scalar fields [41]. It was shown 
that cosmological model with cold dark matter and such types of the scalar field (the QCDM-model) agrees slightly better with the now accessible observable data than the $\Lambda$ CDM-model. But difference of quantitative merits of appropriateness is not large enough to pick out one of them at the confidence level of $1 \sigma$. Since the degeneracies between model parameters of dark energy and cosmological parameters $[16,20,26,28,47]$ exist for the background dynamics, the complete analysis of linear density perturbations in both dark matter and dark energy components is important for the improvement of dark energy observational tests. Among a large number of free quintessence parameters and unknown initial values of quintessence perturbation modes there are few models for which the evolution of perturbations has been studied. The general conclusion is that magnitudes of dark energy density perturbations on scales smaller than horizon are essentially lower than the corresponding magnitudes of matter density ones. But the nature of their evolution depends strongly on the scalar field model (its potential, time variation of the EoS parameter, sound speed, etc.), initial conditions, scale of perturbations and gauge (see for example $[3,4,12-14,17,25,29,44])$.

In this respect special attention should be paid to the EoS parameter of dark energy $w_{Q}$, which can be constant or varying in time. The temporal variation of the dark energy EoS parameter is often presented by linear fitting formula with two [10] or three [24] parameters to be estimated. Other functional dependences of $w_{Q}$ on the scale factor or redshift can be found in $[11,27,38]$. Here we study the parametrization of the equation of state, which needs only one additional quantity with clear physical meaning - the adiabatic speed of sound $c_{a}^{2} \equiv \dot{p}_{Q} / c^{2} \dot{\rho}_{Q}$ (the analysis of generalized dark sector components can be found in earlier works $[21,22])$. In general, $c_{a}^{2}$ is the unknown function of time. However, taking into account simplicity we restrict ourselves to $c_{a}^{2}=$ const, so it is regarded only as the second physical parameter defining the equation of state of dark energy (the first one being the present value of $w_{Q}$ ).

In this paper we undertake a comparative analysis of the evolution of gauge-invariant variables of scalar perturbations in a model with non-relativistic matter $\left(p_{M} \ll c^{2} \rho_{M}\right)$ and scalar field which we define by classical Lagrangian with potential constructed for two cases $\left(w_{Q}=\right.$ const and $\left.c_{a}^{2}=0\right)$ in the concordance cosmological models. These cases have been chosen because they allow us to obtain analytical solutions which seems to look very attractive in the world of numerical computations. We assume the adiabatic initial conditions for matter and dark energy scalar perturbations.

\section{BACKGROUND COSMOLOGICAL AND SCALAR FIELD MODELS}

We consider the homogeneous and isotropic flat Universe with the metric of 4 -space

$$
d s^{2}=g_{i j} d x^{i} d x^{j}=c^{2} d t^{2}-a^{2}(t) \delta_{\alpha \beta} d x^{\alpha} d x^{\beta}
$$

$$
=a^{2}(\eta)\left(d \eta^{2}-\delta_{\alpha \beta} d x^{\alpha} d x^{\beta}\right),
$$

where the factor $a(t)$ is the scale factor, normalized to 1 at the current epoch $t_{0}, \eta$ being conformal time $(c d t=a(\eta) d \eta)$. Henceforth we also put $c=1$, so the time variable $t \equiv x_{0}$ has the dimension of length. Here and below the Latin indices $i, j, \ldots$ run from 0 to 3 , the Greek ones - over the spatial part of the metric: $\nu, \mu, \ldots=1,2,3$.

If the Universe is filled with non-relativistic matter (cold dark matter and baryons) and quintessence which interact only gravitationally (minimal coupling), the dynamics of its expansion is completely described by the Einstein equations

$$
R_{i j}-\frac{1}{2} g_{i j} R=8 \pi G\left(T_{i j}^{(M)}+T_{i j}^{(Q)}\right),
$$

where $R_{i j}$ is the Ricci tensor and $T_{i j}^{(M)}, T_{i j}^{(Q)}$ are energymomentum tensors of Matter $(M)$ and Quintessence $(Q)$. If these components interact only gravitationally then each of them satisfy the differential energy-momentum conservation law separately:

$$
T_{j ; i}^{i(M, Q)}=0
$$

(here and below ";" denotes the covariant derivative with respect to the coordinate with the given index in space with the metric $\left.g_{i j}\right)$. For the perfect fluid with density $\rho_{(M, Q)}$ and pressure $p_{(M, Q)}$, related by the equation of state $p_{(M, Q)}=w_{(M, Q)} \rho_{(M, Q)}$ it gives

$$
\dot{\rho}_{(M, Q)}=-3 \frac{\dot{a}}{a} \rho_{(M, Q)}\left(1+w_{(M, Q)}\right)
$$

(here and below a dot over the variable denotes the derivative with respect to the conformal time: " $" \equiv$ $d / d \eta)$. The matter is considered to be non-relativistic, so $w_{M}=0$ and $\rho_{M}=\rho_{M}^{(0)} a^{-3}$ (here and below " 0 " denotes the present values).

We assume the quintessence to be a scalar field $Q(\mathbf{x}, \eta)$ with classical Lagrangian

$$
L=\frac{1}{2} Q_{; i} Q^{; i}-U(Q),
$$

where $U(Q)$ is the field potential. We suppose also the background scalar field to be homogeneous $(Q(\mathbf{x}, \eta)=$ $Q(\eta))$, so its energy density and pressure depend only on time:

$\rho_{Q}(\eta)=\frac{1}{2 a^{2}} \dot{Q}^{2}+U(Q), \quad p_{Q}(\eta)=\frac{1}{2 a^{2}} \dot{Q}^{2}-U(Q)$.

Then the conservation law (2.2) gives a scalar field evolution equation (known as the Klein-Gordon equation)

$$
\ddot{Q}+2 a H \dot{Q}+a^{2} \frac{d U}{d Q}=0,
$$

where $H=\dot{a} / a^{2}$ is the Hubble parameter for any moment of conformal time $\eta$.

We specify the model of quintessence using two thermodynamical parameters: the EoS parameter $w_{Q} \equiv$ 
$p_{Q} / \rho_{Q}$ and the adiabatic speed of sound $c_{a}^{2} \equiv \dot{p}_{Q} / \dot{\rho}_{Q}$. In the general case they are connected by the equation

$$
\frac{d w / d \ln a}{3(1+w)}=w-c_{a}^{2}
$$

(here and below we omit index $Q$ for $w_{Q}$ ). If the time dependence of $w$ is known then $c_{a}^{2}$ is defined unambiguously, if $c_{a}^{2}$ is determined then the initial value $w_{0}$ must be defined additionaly, so, the EoS parameter has 2 degrees of freedom: a function and a constant. For other parametrizations see $[11,27,38]$. Since the constraints for time dependence of $w$ or $c_{a}^{2}$ are not established well we consider two simple cases: $w=$ const and $c_{a}^{2}=$ const. In the first case $c_{a}^{2}=w$ and in the second one $1+$ $w(a)=\left(1+c_{a}^{2}\right)\left(1+w_{0}\right) /\left(1+w_{0}-\left(w_{0}-c_{a}^{2}\right) a^{3\left(1+c_{a}^{2}\right)}\right)$. This equation has obvious asymptotical behaviour: when $a \rightarrow 0 w \rightarrow c_{a}^{2}$ and when $a \rightarrow \infty w \rightarrow-1$. So, in the early epoch the dark energy mimics dust matter $(w \approx 0)$ for $c_{a}^{2}=0$ or radiation $(w \approx 1 / 3)$ for $c_{a}^{2}=1 / 3$. In future such scalar field will mimic cosmological constant $(w \approx-1)$. The time dependences of EoS parameter for both cases are shown in Fig. 1. The equation (2.3) has the analytical solutions for two cases:

$$
\begin{aligned}
& \text { - } w=\text { const: } \rho_{Q}(a)=\rho_{Q}^{(0)} a^{-3(1+w)} \text { and } \\
& \text { - } c_{a}^{2}=0: \rho_{Q}(a)=\rho_{Q}^{(0)}\left[\left(1+w_{0}\right) a^{-3}-w_{0}\right],
\end{aligned}
$$

so it is possible to simplify formulae and calculations and we will analyse only these two cases now.

If the parametrization of EoS parameter is given, it is possible to apply reverse engineering and construct the fields $Q$ and potentials $U(Q)$. From (2.5) one simply obtains:

$$
Q(a)-Q_{0}= \pm \int_{1}^{a} \frac{\sqrt{\rho_{Q}(1+w)}}{a H}, U(a)=\frac{\rho_{Q}(1-w)}{2} .
$$

If the integral for $Q$ can be expressed via functions that could be inverted to obtain $a\left(Q-Q_{0}\right)$ then $U\left(Q-Q_{0}\right)$ can be easily written in the analytical form.

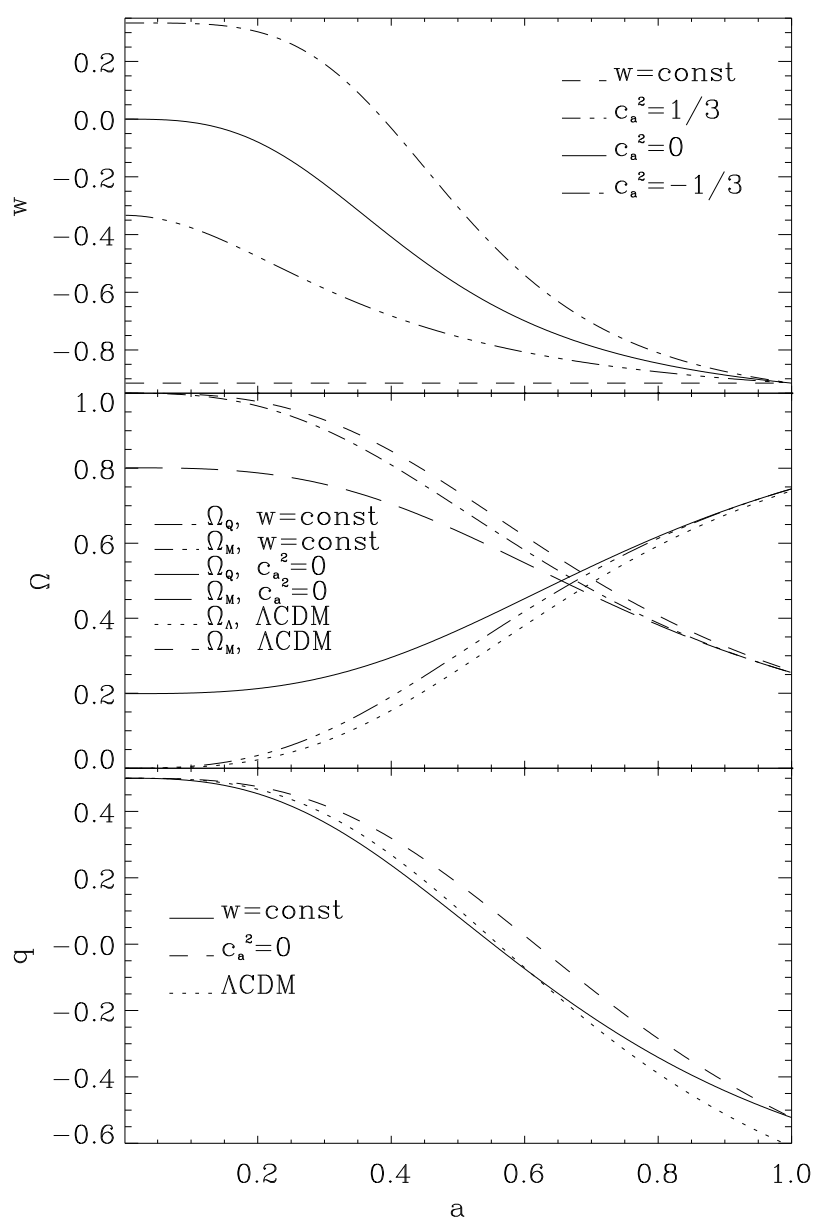

Fig. 1. Top: the dependence of EoS parameter $w$ on scale factor $a$ for $c_{a}^{2}=1 / 3,0,-1 / 3$ and $w=c_{a}^{2}=$ const. Middle: the dynamics of expansion of the homogeneous Universe in the model with non-relativistic matter and quintessential scalar field with $c_{a}^{2}=0$ and $w=c_{a}^{2}=$ const with best fitting cosmological parameters from Spergel et al. (2007) $\left(\Omega_{Q}=0.745, w=-0.915, \Omega_{M}=0.255, h=0.7\right)$ : matter and quintessence densities in units of the critical one. Bottom: the evolution of acceleration parameter. For comparison we show also the corresponding dependences for $\Lambda$ CDM-model with $\Omega_{Q}=0.74, \Omega_{M}=0.26$ and $h=0.73$ (Spergel et al. (2007), Apunevych et al. (2007)).

So, from the Einstein and field equations we deduce the time dependences of the Hubble $H$ and acceleration $q$ parameters as well as the evolution of the scalar field $Q$ and potential $U(Q)$ :

$$
\begin{aligned}
& H=H_{0} a^{-\frac{3}{2}} \sqrt{1-\Omega_{Q}+\Omega_{Q} a^{-3 w}}, \quad q=\frac{1}{2} \frac{1-\Omega_{Q}+(1+3 w) \Omega_{Q} a^{-3 w}}{1-\Omega_{Q}+\Omega_{Q} a^{-3 w}} \\
& Q(a)-Q_{0}= \pm \frac{1}{2 \sqrt{6 \pi G}} \frac{\sqrt{1+w}}{w} \ln \left(\frac{\sqrt{\left(1-\Omega_{Q}\right) a^{3 w}+\Omega_{Q}}-\sqrt{\Omega_{Q}}}{1+\sqrt{\Omega_{Q}}} \frac{1+\sqrt{\left(1-\Omega_{Q}\right) a^{3 w}+\Omega_{Q}}+\sqrt{\Omega_{Q}}}{1-\sqrt{\Omega_{Q}}}\right) \\
& U\left(Q-Q_{0}\right)=\frac{3 H_{0}^{2}}{8 \pi G} \Omega_{Q} \frac{1-w}{2}\left[\operatorname{ch}\left(\sqrt{6 \pi G}\left(Q-Q_{0}\right) \frac{w}{\sqrt{1+w}}\right) \mp \frac{1}{\sqrt{\Omega_{Q}}} \operatorname{sh}\left(\sqrt{6 \pi G}\left(Q-Q_{0}\right) \frac{w}{\sqrt{1+w}}\right)\right]^{2 \frac{1+w}{w}}
\end{aligned}
$$

for $w=$ const and

$$
H=H_{0} a^{-\frac{3}{2}} \sqrt{1+\Omega_{Q} w_{0}-\Omega_{Q} w_{0} a^{3}}, \quad q=\frac{1}{2} \frac{1+w_{0} \Omega_{Q}+2 w_{0} \Omega_{Q} a^{3}}{1+\Omega_{Q} w_{0}-\Omega_{Q} w_{0} a^{3}},
$$




$$
\begin{aligned}
& Q(a)-Q_{0}= \pm \frac{1}{2 \sqrt{6 \pi G}} \sqrt{\frac{\Omega_{Q}\left(1+w_{0}\right)}{1+\Omega_{Q} w_{0}}} \ln \left(\frac{\sqrt{1+\Omega_{Q} w_{0}\left(1-a^{3}\right)}-\sqrt{1+\Omega_{Q} w_{0}}}{\sqrt{1+\Omega_{Q} w_{0}\left(1-a^{3}\right)}+\sqrt{1+\Omega_{Q} w_{0}}} \frac{\sqrt{1+\Omega_{Q} w_{0}}}{1-\sqrt{1+\Omega_{Q} w_{0}}}\right) \\
& U\left(Q-Q_{0}\right)= \frac{3 H_{0}^{2}}{8 \pi G} \frac{\Omega_{Q}\left(1+w_{0}\right)}{2}\left[\operatorname{ch}\left(\sqrt{6 \pi G}\left(Q-Q_{0}\right) \sqrt{\frac{1+\Omega_{Q} w_{0}}{\Omega_{Q}\left(1+w_{0}\right)}}\right)\right. \\
&\left.\mp \frac{1}{\sqrt{1+\Omega_{Q} w_{0}}} \operatorname{sh}\left(\sqrt{6 \pi G}\left(Q-Q_{0}\right) \sqrt{\frac{1+\Omega_{Q} w_{0}}{\Omega_{Q}\left(1+w_{0}\right)}}\right)\right]^{2}-\frac{3 H_{0}^{2}}{8 \pi G} \Omega_{Q} w_{0}
\end{aligned}
$$

for $c_{a}^{2}=0$.

For both models there are 2 independent solutions for the field (the growing one corresponds to sign "+" and the decaying one to sign "-") and 2 symmetrical with respect to $Q-Q_{0}$ potentials exist. However, the physical consequences of both these solutions are the same [40], so from now we restrict ourselves only to the growing one. The variance of scalar field potentials was presented in [11]. The potential for $w=$ const can be also found in $[36,40]$, the potential for $c_{a}^{2}=0$ belongs generally to the family of double exponential potentials (with an additional constant term), but they both differ from the physicallymotivated ones, for which the evolution of scalar linear perturbations was studied by other authors.

We must note that the asymptotic behaviour at $a \rightarrow 0$ of the expansion rate $H(a)$ and acceleration parameter $q(a)$ in both cases is the same and similar to that in the $\Lambda$ CDM-model: $H \propto a^{-3 / 2}, q \rightarrow 1 / 2$. In the current epoch the parameters of expansion dynamics are the same $\left(H_{0}\right.$ and $\left.q_{0}=\left(1+3 w \Omega_{Q}\right) / 2\right)$ for both models. But their asymptotic behaviour at $a \rightarrow \infty$ is different: in $w=$ const quintessence $H \rightarrow H_{0} a^{-\frac{3}{2}(1+w)} \sqrt{\Omega_{Q}}$, $q \rightarrow(1+3 w) / 2, \rho_{Q} \rightarrow 0$ and in $c_{a}^{2}=0$ quintessence $H \rightarrow H_{0} \sqrt{-w_{0} \Omega_{Q}}, q \rightarrow-1, \rho_{Q} \rightarrow-w_{0} \rho_{Q}^{(0)}$. The energy densities of both fields evolve similarly but have different asymptotic regimes: in the quintessence with $w=$ const $\rho_{Q} / \rho_{M}=\Omega_{Q} a^{-3 w} /\left(1-\Omega_{Q}\right)$ always while in the $c_{a}^{2}=0$ quintessence at $a \rightarrow 0 \rho_{Q} / \rho_{M} \rightarrow\left(1+w_{0}\right) \Omega_{Q} /\left(1-\Omega_{Q}\right)$ and at $a \rightarrow \infty \rho_{Q} / \rho_{M} \rightarrow-w_{0} \Omega_{Q} a^{3} /\left(1-\Omega_{Q}\right)$. So, the scalar field with $c_{a}^{2}=0$ behaves as cold dark matter at the early epoch and will mimic the cosmological constant in the far future.

The different asymptotic behaviour of these fields is caused by their intrinsic properties. In the $w=$ const quintessence the negative pressure stiffly follows its energy density and their relation is always constant. In the $c_{a}^{2}=0$ quintessence the negative pressure is always constant: $p_{Q}=3 H_{0}^{2} w_{0} \Omega_{Q} / 8 \pi G$. So, it is insignificant in the early epoch when $a \rightarrow 0$ and $\rho_{Q} \rightarrow \infty$ for the model of the Universe filled only with dust matter and quintessential dark energy, and important in the late one when $w \rightarrow-1$.

The dynamics of expansion of homogeneous Universe in the model with non-relativistic matter and quintessential scalar field with $c_{a}^{2}=0$ and $w=c_{a}^{2}=$ const is shown in Fig. 1. For both models we assume best fitting cosmological parameters from [43] $\left(\Omega_{Q}=0.745\right.$, $\left.w=w_{0}=-0.915, \Omega_{M}=0.255, h=0.7\right)$. For comparison we show also the corresponding dependences in $\Lambda$ CDM-model with $\Omega_{\Lambda}=0.74, \Omega_{M}=0.26$ and $h=0.73$ $[1,43]$.

We have constructed the potentials of quintessential scalar fields with $w=$ const [40] and $c_{a}^{2}=0$ for the QCDM cosmological model with best fitting parameters obtained from WMAP and SNIa data [43]. The evolution of the fields $Q(a)$, potentials $U(a)$ and rolling down of the fields $Q$ to the minimum which is located at $Q \rightarrow \infty$ $(a \rightarrow \infty)$ are shown in Fig. 2. The discussion of the influence of parameter determination uncertainties on a potential of field with $w=$ const can be found in [40].

So, the difference of the homogeneous Universe expansion dynamics in $\Lambda$ CDM- and such QCDM-models is too small to discriminate them using the avialable datasets. That is why in the next sections we will analyse the linear stage of growth of scalar perturbations of matter and dark energy. For this we will use gauge-invariant approach developed by $[2,15,23]$.

\section{EVOLUTION OF SCALAR PERTURBATIONS}

For the analysis of scalar linear perturbations the conformal-Newtonian gauge with space-time metric

$$
\begin{aligned}
& d s^{2}=a^{2}(\eta)\left[(1+2 \Psi(\mathbf{x}, \eta)) d \eta^{2}\right. \\
& \left.-(1+2 \Phi(\mathbf{x}, \eta)) \delta_{\alpha \beta} d x^{\alpha} d x^{\beta}\right]
\end{aligned}
$$

is convenient. Here $\Psi(\mathbf{x}, \eta)$ and $\Phi(\mathbf{x}, \eta)$ are gaugeinvariant perturbations of a metric [2] called Bardeen's potentials. If proper anisotropy of the medium equals zero then $\Psi(\mathbf{x}, \eta)=-\Phi(\mathbf{x}, \eta)$. Dust matter and scalar fields have this property [23]. In the linear perturbation theory the Fourier decomposition is used, so spatial dependences of all variables can be substituded by the corresponding Fourier amplitudes. For example, $\Psi(\mathbf{x}, \eta) \rightarrow \Psi(k, \eta)$, where $k$ is wave number. Henceforth, telling about the metric $\Psi(\mathbf{x}, \eta)$, matter density $\delta^{(M)}(\mathbf{x}, \eta) \equiv\left(\rho_{M}(\mathbf{x}, \eta)-\bar{\rho}_{M}(\eta)\right) / \bar{\rho}_{M}(\eta)$, its peculiar velocity $V^{(M)}(\mathbf{x}, \eta)$, scalar field $\delta Q(\mathbf{x}, \eta) \equiv Q(\mathbf{x}, \eta)-\bar{Q}(\eta)$, its energy density perturbations $\delta^{(Q)}(\mathbf{x}, \eta) \equiv\left(\rho_{Q}(\mathbf{x}, \eta)-\right.$ 
$\left.\bar{\rho}_{Q}(\eta)\right) / \bar{\rho}_{Q}(\eta)$ etc. we mean their Fourier amplitudes $\Psi(k, \eta), \delta^{(M)}(k, \eta), V^{(M)}(k, \eta), \delta Q(k, \eta), \delta^{(Q)}(k, \eta)$, etc. The metric $(\Psi(k, \eta))$, matter density and velocity perturbations $\left(\delta^{(M)}(k, \eta), V^{(M)}(k, \eta)\right)$ as well as scalar field perturbations $\left(\delta Q(k, \eta), \delta^{(Q)}(k, \eta), V^{(Q)}(k, \eta)\right)$ in the conformal-Newtonian gauge are gauge-invariant variables [23]. The energy density and velocity perturbations of quintessence, $\delta^{(Q)}$ and $V^{(Q)}$, are connected with the perturbation of field variable $\delta Q$ in the following way:

$$
\begin{aligned}
\delta^{(Q)} & =(1+w)\left(\frac{\dot{\delta}}{\dot{Q}}-\Psi+\frac{a^{2} \delta Q}{\dot{Q}^{2}} \frac{d U}{d Q}\right), \\
V^{(Q)} & =\frac{k \delta Q}{\dot{Q}} .
\end{aligned}
$$

Other non-vanishing gauge-invariant perturbations of the scalar field are isotropic pressure perturbation

$$
\pi_{L}^{(Q)}=\frac{1+w}{w}\left(\frac{\dot{\delta Q}}{\dot{Q}}-\Psi-\frac{a^{2} \delta Q}{\dot{Q}^{2}} \frac{d U}{d Q}\right)
$$

and intrinsic entropy

$$
\Gamma^{(Q)}=\pi_{L}^{(Q)}-\frac{c_{a}^{2}}{w} \delta^{(Q)} .
$$

The density perturbation of any component in the conformal-Newtonian gauge $D_{s} \equiv \delta$, which is gaugeinvariant variable, is related to other gauge-invariant variables of density perturbations $D$ and $D_{g}$ as:
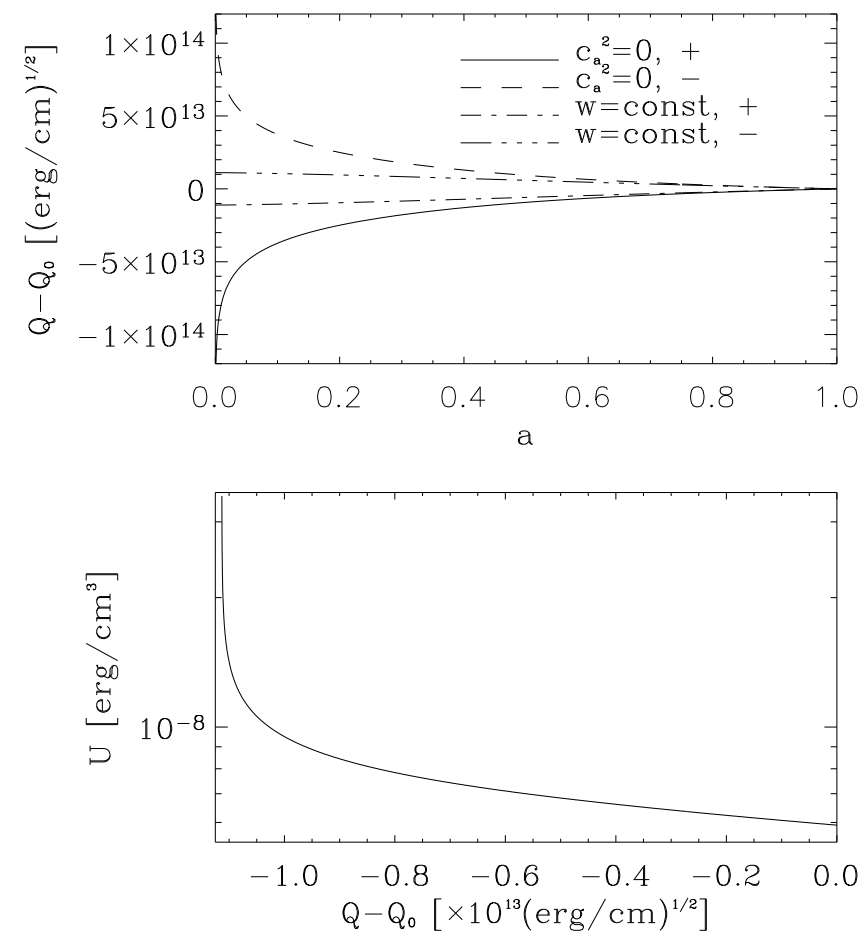

$$
D=D_{g}+3(1+w)\left(\Psi+\frac{\dot{a}}{a} \frac{V}{k}\right)=D_{s}+3(1+w) \frac{\dot{a}}{a} \frac{V}{k},
$$

where $D_{s}, D, D_{g}$ and $V$ correspond to either $M$ - or $Q$-component. Here $D_{g}$ is the density perturbation in the rest frame in which the fluctuations of the curvature scalar of the constant time hypersurface vanish and $D$ corresponds to the rest frame in which the 4-velocity is orthogonal to constant time hypersurface [23].

The intrinsic entropy of quintessence $\Gamma^{(Q)}$ can be presented via gauge-invariant $Q$-perturbations as follows:

$$
w \Gamma^{(Q)}=\left(1-c_{a}^{2}\right) D^{(Q)}
$$

This equation shows that the intrinsic entropy for scalar perturbations of quintessence with $c_{a}^{2} \neq 1$ is non-zero when proper energy density perturbation $D^{(Q)}$ (measured in synchronous comoving gauge) of quintessence is non-vanishing. In the first case $\left(w=\right.$ const) $w \Gamma^{(Q)}=$ $(1-w) D^{(Q)}$, in the second one $\left(c_{a}^{2}=0\right) w \Gamma^{(Q)}=D^{(Q)}$. In the case of perturbed quintessence dissipative processes generate entropic perturbations, so we have the sound speed $c_{s}^{2}$ defined by a more general relation: $c_{s}^{2} \equiv$ $\delta p_{Q} / \delta \rho_{Q}$. The intrinsic entropy perturbation can be presented in the form: $w \Gamma^{(Q)} \equiv\left(c_{s}^{2}-c_{a}^{2}\right) D^{(Q)}[23]$. For the scalar fields with classical Lagrangian $c_{s}^{2}=1[18,45]$.
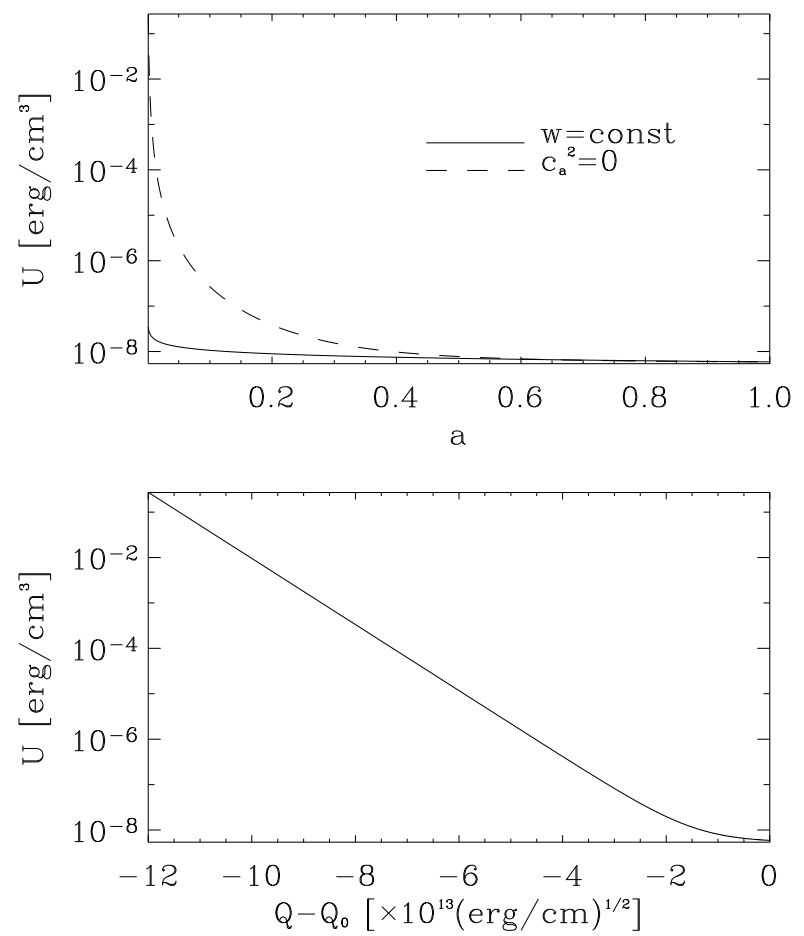

Fig. 2. Top: the evolution of fields $Q(a)$ (left), potentials $U(a)$ (right) for quintessence with $w=$ const and $c_{a}^{2}=0$. Bottom: rolling down of the fields $Q$ to the minimum $U(Q)=0$ which is located at $Q \rightarrow \infty(a \rightarrow \infty)$ for cases of $w=$ const (left) and $c_{a}^{2}=0$ (right). 


\section{A. Evolution equations}

Evolution equation for scalar field perturbation $\delta Q(k, \eta)$ can be obtained either from Lagrange-Euler equation or from energy-momentum conservation law $T_{0 ; i}^{i}{ }^{(Q)}=0$ :

$$
\ddot{\delta Q}+2 a H \dot{\delta Q}+\left(k^{2}+a^{2} \frac{d^{2} U}{d Q^{2}}\right) \delta Q
$$

$$
+2 a^{2} \frac{d U}{d Q} \Psi-4 \dot{\Psi} \dot{Q}=0
$$

Thus, the evolution of quintessence perturbation depends on field model $(U(Q))$, gravitational potential $\Psi$, expansion rate of the Universe $H$ and the scale of perturbation $k$.

The linearised Einstein equations for gauge-invariant perturbations of metric and energy-momentum tensor components are

$$
\begin{aligned}
& D_{g}^{\prime(Q)}+\frac{3}{a}(1-w) D_{g}^{(Q)}+\left(\frac{k}{a^{2} H}+9 \frac{\left(1-c_{a}^{2}\right) H}{k}\right)(1+w) V^{(Q)}+9(1+w)\left(1-c_{a}^{2}\right) \frac{\Psi}{a}=0, \\
& V^{\prime(Q)}-\frac{2}{a} V^{(Q)}-4 \frac{k \Psi}{a^{2} H}-\frac{k}{a^{2} H} \frac{D_{g}^{(Q)}}{1+w}=0, \\
& \Psi^{\prime}+\frac{\Psi}{a}-\frac{4 \pi G}{H}\left(\bar{\rho}_{M} \frac{V^{(M)}}{k}+\bar{\rho}_{Q}(1+w) \frac{V^{(Q)}}{k}\right)=0 .
\end{aligned}
$$

Here and below a prime denotes the derivative with respect to the scale factor $a$. The conservation equations for matter density and velocity perturbations $\delta T_{j ; i}^{i(M)}=0$ in terms of the gauge-invariant variables $D_{g}^{(M)}$ and $V^{(M)}$ are as follows:

$$
\begin{aligned}
& D_{g}^{\prime(M)}+\frac{k V^{(M)}}{a^{2} H}=0, \\
& V^{\prime(M)}+\frac{V^{(M)}}{a}-\frac{k \Psi}{a^{2} H}=0 .
\end{aligned}
$$

They are connected with the dark energy ones only via $\Psi$ and are the same for both models of quintessence.

So, in each case we have the system of 5 first-order ordinary differential equations for 5 unknown functions $\Psi(k, a)$, $D_{g}^{(M)}(k, a), V^{(M)}(k, a), D_{g}^{(Q)}(k, a)$ and $V^{(Q)}(k, a)$. From these systems of equations it is easy to obtain the systems of 2 second-order ordinary differential equations for 2 unknown functions $\Psi(k, a)$ and $\delta Q(k, a)$ :

$$
\begin{gathered}
\Psi^{\prime \prime}+\left(\frac{7}{2}-\frac{3}{2} w \Omega_{Q} a^{-3(1+w)} \frac{H_{0}^{2}}{H^{2}}\right) \frac{\Psi^{\prime}}{a}+\frac{3}{2}(1-w) \Omega_{Q} a^{-3(1+w)} \frac{H_{0}^{2}}{H^{2}} \frac{\Psi}{a^{2}} \\
-a^{-\frac{3}{2}(1+w)} \frac{H_{0}}{H} \sqrt{6 \pi G \Omega_{Q}(1+w)} \frac{2 a \delta Q^{\prime}+3(1-w) \delta Q}{2 a^{2}}=0 \\
\delta Q^{\prime \prime}+\left(\frac{5}{2}-\frac{3}{2} w \Omega_{Q} a^{-3(1+w)} \frac{H_{0}^{2}}{H^{2}}\right) \frac{\delta Q^{\prime}}{a}+\left(\frac{k^{2}}{a^{4} H^{2}}+\frac{9(1-w)(2+w)}{4 a^{2}}+\frac{9 w(1-w)}{4 a^{2}} \Omega_{Q} a^{-3(1+w)} \frac{H_{0}^{2}}{H^{2}}\right) \delta Q \\
-a^{-\frac{3}{2}(1+w)} \frac{H_{0}}{H} \sqrt{\frac{3}{8 \pi G} \Omega_{Q}(1+w)} \frac{4 a \Psi^{\prime}+3(1-w) \Psi}{a^{2}}=0
\end{gathered}
$$

for $w=$ const or

$$
\begin{aligned}
& \Psi^{\prime \prime}+\left(\frac{7}{2}-\frac{3}{2} w_{0} \Omega_{Q} \frac{H_{0}^{2}}{H^{2}}\right) \frac{\Psi^{\prime}}{a}+\frac{3}{2}\left(1+w_{0}-2 w_{0} a^{3}\right) \Omega_{Q} a^{-3} \frac{H_{0}^{2}}{H^{2}} \frac{\Psi}{a^{2}}-a^{-\frac{3}{2}} \frac{H_{0}}{H} \sqrt{6 \pi G \Omega_{Q}\left(1+w_{0}\right)} \\
& \times \frac{2 a \delta Q^{\prime}+3 \delta Q}{2 a^{2}}=0 \\
& \delta Q^{\prime \prime}+\left(\frac{5}{2}-\frac{3}{2} w_{0} \Omega_{Q} \frac{H_{0}^{2}}{H^{2}}\right) \frac{\delta Q^{\prime}}{a}+\left(\frac{k^{2}}{a^{4} H^{2}}+\frac{9}{2 a^{2}}+\frac{9}{4 a^{2}} w_{0} \Omega_{Q} \frac{H_{0}^{2}}{H^{2}}\right) \delta Q-a^{-\frac{3}{2}} \frac{H_{0}}{H} \sqrt{\frac{3}{8 \pi G} \Omega_{Q}\left(1+w_{0}\right)} \\
& \times \frac{4 a \Psi^{\prime}+3 \Psi}{a^{2}}=0
\end{aligned}
$$

for $c_{a}^{2}=0$. 
Using their solutions (four fundamental once) for $\Psi(k, a)$ and $Q(k, a)$ and the constraint equation

$$
-k^{2} \Psi=4 \pi G a^{2}\left(\bar{\rho}_{M} D^{(M)}+\bar{\rho}_{Q} D^{(Q)}\right),
$$

it is possible to find the values of $D_{g}^{(M)}(k, a), V^{(M)}(k, a)$, $D_{g}^{(Q)}(k, a), V^{(Q)}(k, a)$ and $\Gamma^{(Q)}(k, a)$. The equations (3.21)-(3.22) can be substituted by one second-order equation

$$
D_{g}^{\prime \prime(M)}+(2-q) \frac{D_{g}^{\prime(M)}}{a}+\frac{k^{2}}{a^{4} H^{2}} \Psi=0 .
$$

The systems of equations (3.23)-(3.24) and (3.25)(3.26) describe the evolution of perturbations of gravitational $\Psi$ and quintessentional $\delta Q$ fields and their coupling. A few important conclusions can be deduced from the qualitative analysis of these systems:

- The coupling of $\Psi$ - and $\delta Q$-field is modulated by the value $\sqrt{\Omega_{Q}(1+w)}$ for $w=$ const and $\sqrt{\Omega_{Q}\left(1+w_{0}\right)}$ for $c_{a}^{2}=0$. So, if $w=w_{0}=-1$ then both fields evolve independently. Since observational data prefer current $w$ close to -1 their coupling is weak.

- Evolution of $\delta Q$-field depends on the relation of scale of perturbation to horizon explicitly while the dependence of $\Psi$-field is implicit (through the latter).

- The system of equations for $w$-quintessence (3.23)(3.24) allows the asymptotic behaviour $\Psi \rightarrow$ const, $\delta Q \rightarrow$ const when $a \rightarrow \infty$ with the relation between them $\delta Q=\Psi / \sqrt{6 \pi G(1+w)}$.

- The system of equations for $c_{a}^{2}$-quintessence (3.25)-(3.26) allows the asymptotic behaviour $\Psi \rightarrow$ const, $\delta Q \rightarrow$ const when $a \rightarrow 0$ with the initial relation between them $\delta Q=$ $\sqrt{\Omega_{Q}\left(1+w_{0}\right) / 6 \pi G\left(1+\Omega_{Q} w_{0}\right)} \Psi$.

- From equation (3.28) it follows that $D_{g}^{(M)} \approx$ const for superhorizon perturbations $\left(k \ll a^{2} H\right)$. If $\Psi=$ const and $q=1 / 2$ at $a \rightarrow 0$ then either $D_{g}^{(M)}=$ const $-2 a k^{2} \Psi / 3 H_{0}^{2}\left(1-\Omega_{Q}\right)$ for $w=$ const or $D_{g}^{(M)}=$ const $-2 a k^{2} \Psi / 3 H_{0}^{2}\left(1+\Omega_{Q} w_{0}\right)$ for $c_{a}^{2}=0$ and for $\Psi<0$ it begins to grow slowly from the constant value. The decay of $\Psi$ and transition from deceleration to acceleration slow down the growth of $D_{g}^{(M)}$.

\section{B. Initial conditions}

A stady of the background dynamics presented in the previous section has shown that both QCDM-models are matter-dominated in the early Universe (Fig. 1). In the QCDM-model with $w=$ const the ratio $\rho_{M} / \rho_{Q} \rightarrow \infty$ when $a \rightarrow 0$, while in the QCDM-model with $c_{a}^{2}=0$ $\rho_{M} / \rho_{Q} \rightarrow\left(1-\Omega_{Q}\right) /\left(1+w_{0}\right) \Omega_{Q}$ and $w \rightarrow 0$ when $a \rightarrow 0$. The adiabatic growing mode of perturbation in the non-relativistic matter-dominated Universe can be specified by the condition $\Psi=$ const $(\dot{\Psi}=0)$. Adiabaticity condition in two-component model $\left(S_{M: Q} \equiv D_{g}^{(M)}-\right.$ $\left.D_{g}^{(Q)} /(1+w)=0[14]\right)$ gives $D_{g}^{(M)}=D_{g}^{(Q)} /(1+w)$. These conditions, constraint equations written for hypersurface $\eta_{\text {init }} \ll \eta_{0}\left(a_{\text {init }} \ll 1\right)$ and the analytic asymptotic solutions (see next subsection) lead to the following adiabatic initial conditions:

$$
\begin{aligned}
V_{\text {init }}^{(Q)} & =\frac{2}{3} \frac{k}{H_{0}} \frac{\Psi_{\text {init }}}{\sqrt{1-\Omega_{Q}}} \sqrt{a_{\text {init }}} \\
D_{g}^{(Q)}{ }_{\text {init }} & =-5(1+w) \Psi_{\text {init }} \\
V_{\text {init }}^{(M)} & =\frac{2}{3} \frac{k}{H_{0}} \frac{\Psi_{\text {init }}}{\sqrt{1-\Omega_{Q}}} \sqrt{a_{\text {init }}} \\
D_{g}^{(M)}{ }_{\text {init }} & =-5 \Psi_{\text {init }}
\end{aligned}
$$

for $w=$ const and

$$
\begin{aligned}
V_{\text {init }}^{(Q)} & =\frac{2}{3} \frac{k}{H_{0}} \frac{\Psi_{\text {init }}}{\sqrt{1+\Omega_{Q} w_{0}}} \sqrt{a_{\text {init }}}, \\
D_{g}^{(Q)}{ }_{\text {init }} & =-5 \Psi_{\text {init }}, \\
V_{\text {init }}^{(M)} & =\frac{2}{3} \frac{k}{H_{0}} \frac{\Psi_{\text {init }}}{\sqrt{1+\Omega_{Q} w_{0}}} \sqrt{a_{\text {init }}}, \\
D_{g}^{(M)}{ }_{\text {init }} & =-5 \Psi_{\text {init }}
\end{aligned}
$$

for $c_{a}^{2}=0$.

Therefore, the growing mode of adiabatic perturbations in two-component (non-relativistic matter and quintessence) medium is defined by the single value initial gravitational potential $\Psi_{\text {init }}$.

Since the non-adiabatic initial perturbations are strongly constrained by the WMAP data, in this paper we restrict ourselves only to adiabatic initial conditions.

\section{Asymptotic and numerical solutions}

In order to analyse the evolution of gauge-invariant variables of matter and quintessence perturbations we must solve the system of equations (3.18)-(3.20) together with (3.21)-(3.22) numerically for initial conditions (3.29)-(3.32) or (3.33)-(3.36), respectively. But before we propose the analysis of these systems of equations in the early epoch $(a \ll 1)$, for which the analytical solutions are known. So, the system of equations (3.25)(3.26) for $a \ll 1$ can be simplified as

$$
\begin{aligned}
& \Psi^{\prime \prime}+\frac{7}{2} \frac{\Psi^{\prime}}{a}+\frac{3}{2} \frac{\Omega_{Q}\left(1+w_{0}\right)}{1+\Omega_{Q} w_{0}} \frac{\Psi}{a^{2}}-\sqrt{\frac{6 \pi G \Omega_{Q}\left(1+w_{0}\right)}{1+\Omega_{Q} w_{0}}} \\
& \times \frac{2 a \delta Q^{\prime}+3 \delta Q}{2 a^{2}}=0, \\
& \delta Q^{\prime \prime}+\frac{5}{2} \frac{\delta Q^{\prime}}{a}+\frac{9}{2 a^{2}} \delta Q-\sqrt{\frac{3}{8 \pi G} \frac{\Omega_{Q}\left(1+w_{0}\right)}{1+\Omega_{Q} w_{0}}} \\
& \times \frac{4 a \Psi^{\prime}+3 \Psi}{a^{2}}=0 .
\end{aligned}
$$

This system of equations has 4 fundamental solutions, so it is possible to write the general solution in the form: 


$$
\begin{aligned}
\Psi= & C_{1}+\frac{C_{2}}{a^{\frac{5}{2}}}+C_{3} a^{-\frac{3}{4}\left(1+\sqrt{\frac{\Omega_{Q} w_{0}+8 \Omega_{Q}-7}{1+\Omega_{Q} w_{0}}}\right)}+C_{4} a^{-\frac{3}{4}\left(1-\sqrt{\frac{\Omega_{Q} w_{0}+8 \Omega_{Q}-7}{1+\Omega_{Q} w_{0}}}\right)}, \\
\delta Q= & \frac{1}{\sqrt{6 \pi G}} \sqrt{\frac{\Omega_{Q}\left(1+w_{0}\right)}{1+\Omega_{Q} w_{0}}}\left[C_{1}+\frac{3}{2} \frac{C_{2}}{a^{\frac{5}{2}}}-\frac{1+\Omega_{Q} w_{0}}{\Omega_{Q}\left(1+w_{0}\right)} \sqrt{\frac{\Omega_{Q} w_{0}+8 \Omega_{Q}-7}{1+\Omega_{Q} w_{0}}} \times\right. \\
& \left.\left(C_{3} a^{-\frac{3}{4}\left(1+\sqrt{\frac{\Omega_{Q} w_{0}+8 \Omega_{Q}-7}{1+\Omega_{Q} w_{0}}}\right)}-C_{4} a^{-\frac{3}{4}\left(1-\sqrt{\frac{\Omega_{Q} w_{0}+8 \Omega_{Q}-7}{1+\Omega_{Q} w_{0}}}\right)}\right)\right] .
\end{aligned}
$$
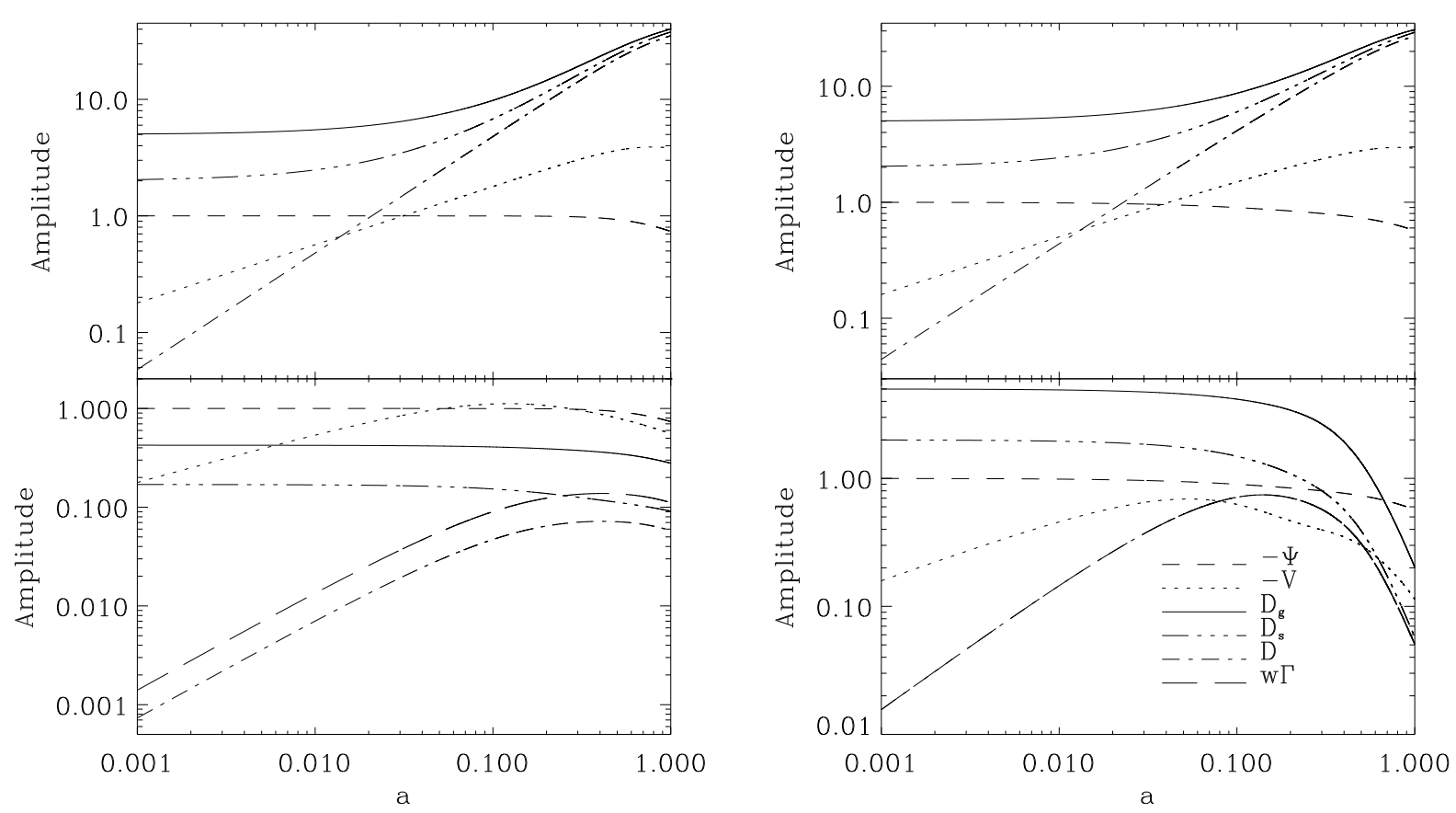

Fig. 3. The evolution of gauge-invariant amplitudes of perturbations in matter (top) and quintessence (bottom) for two models of quintessence: $w=$ const (left column) and $c_{a}^{2}=0$ (right column). The corresponding scale of perturbations is $k=0.00 \mathrm{Mpc}^{-1}$ and the cosmological parameters are $\Omega_{Q}=0.745, w=-0.915, \Omega_{M}=0.255, h=0.7$.

The first two solutions noted by the constants of integration $C_{1}$ and $C_{2}$ are well known growing and decaying modes of adiabatic perturbations in the dust matterdominated Universe. The next two solutions, noted by the constants of integration $C_{3}$ and $C_{4}$, are due to possible entropy initial conditions and intrinsic non-vanishing entropy of quintessence. Indeed, the condition $\Gamma^{(Q)}=0$ leads to 1 second-order equation which has two dust-like fundamental solutions:

$\Psi=\tilde{C}_{1}+\tilde{C}_{2} a^{-\frac{5}{2}} \quad$ and $\quad D_{s}^{(Q)}=-2\left(\tilde{C}_{1}-\frac{3}{2} \tilde{C}_{2} a^{-\frac{5}{2}}\right)$

For the quintessence with $w=$ const the solutions for $\Psi$ are the same and $D_{s}^{(Q)}=-2(1+w)\left(\tilde{C}_{1}-\frac{3}{2} \tilde{C}_{2} a^{-\frac{5}{2}}\right)$.

The quantities $D_{g}^{(M)}(k, a), V^{(M)}(k, a)$ and $V^{(Q)}(k, a)$ can be found using equations (3.15)-(3.22) and (3.27). The relations between them are presented in the previ- ous subsection as a set of initial data (3.29)-(3.36).

We have integrated numerically the systems of equations (3.18)-(3.20) for $w=$ const and for $c_{a}^{2}=0$ together with (3.21)-(3.22) for adiabatic initial conditions (3.29)-(3.32) and (3.33)-(3.36) using the publicly available code DVERK ${ }^{1}$. We assumed $a_{\text {init }}=10^{-10}$ and integrated up to $a=1$. The evolution of perturbations is scale dependent, so we performed calculations for $k=0.0001,0.001,0.01$ and $0.1 \mathrm{Mpc}^{-1}$ for the cosmological model with the parameters $\Omega_{Q}=0.745, w=-0.915$, $\Omega_{M}=0.255, h=0.7$. The evolution of gauge-invariant variables of matter perturbations $D_{g}^{(M)}, D_{s}^{(M)}, D^{(M)}$, $V^{(M)}$ for two scales $k=0.001$ and $0.01 \mathrm{Mpc}^{-1}$ is shown in top panels of Fig. 3 and Fig. 4. In the bottom panels the analogical gauge-invariant variables of quintessence perturbations $\left(D_{g}^{(Q)}, D_{s}^{(Q)}, D^{(Q)}, V^{(Q)}, w \Gamma^{(Q)}\right)$ are presented (for $c_{a}^{2}=0$ the curves $w \Gamma^{(Q)}$ and $D^{(Q)}$ overlap). The evolution of gauge-invariant gravitational potential

\footnotetext{
${ }^{1}$ It was created by T. E. Hull, W. H. Enright, K. R. Jackson in 1976 and is available at http://www.cs.toronto.edu/NA/dverk.f.gz
} 

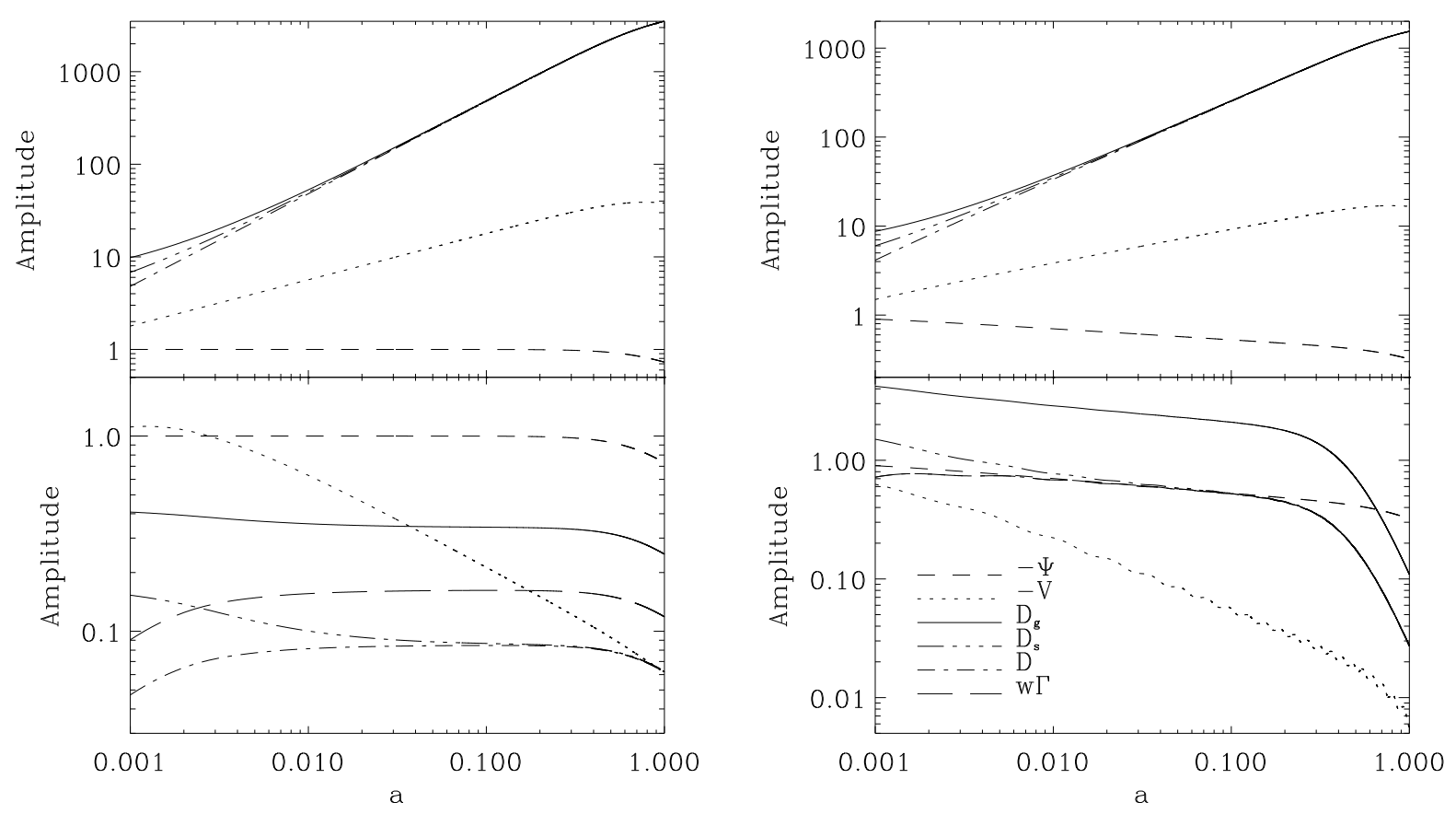

Fig. 4. The same as in Fig. 3 for scale $k=0.01 \mathrm{Mpc}^{-1}$.

\section{DISCUSSION}

From the top panels of Fig. 3, 4 it follows that the magnitudes of the adiabatic matter density perturbations grow similarly in both models (and alike in the $\Lambda$ CDMmodel), but time dependences of magnitudes of the adiabatic quintessence energy density perturbations are more varied (bottom panels of the same figures): gaugeinvariant variables $D_{g}^{(Q)}$ and $D_{s}^{(Q)}$ decay from the initial constant value after the particle horizon entry while $D^{(Q)}$ and $V^{(Q)}$ grow at an early stage before the horizon entry and decay after that - in the quintessence-dominated epoch when the gravitational potential starts to decay. The perturbation shown in Fig. 3 enters the particle horizon $(\eta(a)=\pi / k)$ at $a \approx 0.03$ for $w$-quintessence and at $a \approx 0.04$ for $c_{a}^{2}$-quintessence. The perturbation shown in Fig. 4 enters the particle horizon at $a \approx 0.0004$ and $a \approx$ 0.0005 for $w$ - and $c_{a}^{2}$-quintessence, respectively. The particle horizon in the current epoch $\left(\eta_{0}\right)$ in the cosmological model with the parameters $\Omega_{Q}=0.745, w=-0.915$, $\Omega_{M}=0.255, h=0.7$ and $w$-quintessence equals $\approx 14970$ Mpc. In the model with $c_{a}^{2}$-quintessence it is $\approx 13810$ Mpc. In the early epoch $D^{(Q)} \propto a$ for both models of quintessence. After the particle horizon entry the amplitudes start to decay slowly in the matter-dominated epoch and decay fast in the quintessence-dominated one. At asymptotic regime for the quintessence model with $c_{a}^{2}=0$ approximately $D^{(Q)} \propto a^{-3}$. In the quintessence model with $w=$ const the transition epoch is extend- ed in time. In Fig. 5 we show the dependences of ratios of quintessence density perturbations to matter density ones in conformal-Newtonian gauge $\left(D_{s}^{(Q)} / D_{s}^{(M)}\right)$ on the scale factor for perturbations with the scales $k=0.0001$, $0.001,0.01$ and $0.1 \mathrm{Mpc}^{-1}$. These curves emphasise the difference of evolution of perturbations in ordinary matter and quintessence as well as the similarity of behaviour of perturbations in two models of quintessence. The magnitudes of quintessence density perturbations in units of matter density perturbations in both models in the current epoch are close although their initial magnitudes differ by one order. The magnitudes of quintessence density perturbations with the scale less than particle horizon are lower than the corresponding magnitudes of matter density perturbations by factor $\approx(23000 k)^{2}$ so that for scale $k=0.01 D_{s}^{(Q)} / D_{s}^{(M)} \approx 2 \times 10^{-5}$. Therefore, on subhorizon scales the quintessential scalar fields are practically smoothed out while the matter clusters.

The tests for choice of the type of dark energy are based on the results of its action on luminous matter and cosmic microwave background. So, the key question is how these types of quintessence affect the growth of matter density perturbations and the time variation of gravitational potential. From top panels of Fig. 3 and 4 we can see that they are more suppressed for $c_{a}^{2}=0$ than for $w=$ const and for perturbations with smaller scale. In order to illustrate this effect in Fig. 6 we present ratios $D^{(M)} a_{\text {init }} / D_{\text {init }}^{(M)} a$ and $\Psi / \Psi_{\text {init }}$ for scales $k=0.0001,0.001,0.01$ and $0.1 \mathrm{Mpc}^{-1}$. We can see that 
scale dependence of suppression of magnitude of matter density perturbations as well as of gravitational potential is strong for the $c_{a}^{2}=0$ quintessence and weak for the $w=$ const one. In the $\Lambda$ CDM-model it is scaleindependent [9]. (In the Einstein - de Sitter model both ratios equal 1 for all times and scales). These ratios are substantial for calculations of magnitude of the matter density power spectrum at different redshifts and the angular power spectrum of CMB temperature fluctuations in the range of scales of the late integrated Sachs-Wolfe effect.

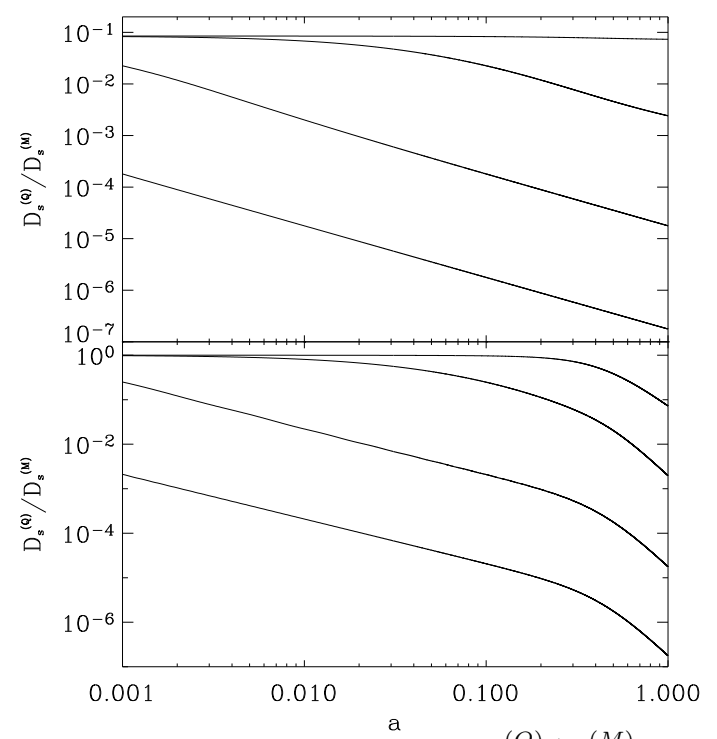

Fig. 5. Dependences of ratios $D_{s}^{(Q)} / D_{s}^{(M)}$ on scale factor for linear perturbations with scales $k=0.0001,0.001,0.01$ and $0.1 \mathrm{Mpc}^{-1}$ (from top to bottom) in the models with non-relativistic matter and quintessence $(w=$ const - top panel, $c_{a}^{2}=0-$ bottom).

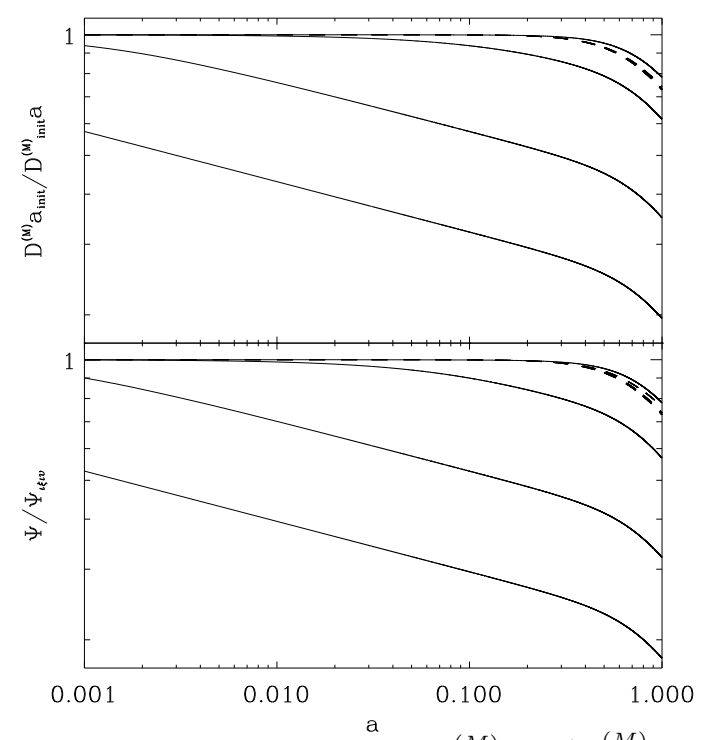

Fig. 6. Evolution of ratios $D^{(M)} a_{\text {init }} / D_{\text {init }}^{(M)} a$ and $\Psi / \Psi_{\text {init }}$ for linear perturbations with scales $k=0.0001,0.001,0.01$ and $0.1 \mathrm{Mpc}^{-1}$ (from top to bottom) in the models with non-relativistic matter and quintessence $\left(c_{a}^{2}=0-\right.$ solid line, $w=$ const - dashed line).
Evolution of quintessence perturbations depends on scalar field model (i.e. its Lagrangian and potential), contents of the Universe, coupling of the quintessence to other components, initial conditions and scale of perturbations [3, 4, 12-14, 29, 44]. Here we have analysed the evolution of scalar matter and quintessence perturbations for potentials of scalar fields with classical Lagrangian constructed to give either $w=$ const or $c_{a}^{2}=0$. Therefore, the results obtained here could be compared with those of other authors only qualitatively. The evolution of EoS parameter in our $c_{a}^{2}=0$-model (Fig. 1) is similar to that of [14]. Despite a different cosmological model and potential of scalar field, the qualitative behaviour of quintessence perturbations is close to the obtained here: $D_{g}^{(Q)}$ is const when the perturbation is outside the particle horizon and decays when it enters the horizon. The growth of magnitude of quintessence density perturbations long before the horizon entry in a synchronous gauge was shown in [12] (models with $w=$ const in Fig. 3). Our results for evolution of gaugeinvariant variable $D^{(Q)}$ (density perturbation in a synchronous gauge) shown in Fig. 3 support this conclusion. (We do not discuss the oscilations at early stage visible in Fig. 3 of [12] because of different initial conditions and background.) The ratios of quintessence ( $w=$ const, $c_{s}^{2}=1$ ) density perturbations to matter density ones in a synchronous gauge are shown in Fig. 1 of [4] for $k=0.01 \mathrm{~h}^{-1} \mathrm{Mpc}^{-1}$. Presented here in Fig. 5 analogical ratios in the conformal-Newtonian gauge are similar. The conclusion about anti-correlation between perturbations of the matter and quintessence has been made in [17] and [30] on the basis of a study of their evolution in the matter rest frame. [4] and [45] noted this effect too. Recalculation of the frame-dependent variables to gauge-invariant ones will - in our belief - remove such a discrepancy.

\section{CONCLUSION}

The dynamics of expansion of the Universe and evolution of scalar perturbations are studied for the quintessential scalar fields $Q$ with the classical Lagrangian $L=\frac{1}{2} Q_{; i} Q^{; i}-U(Q)$ satisfying the additional condition $w=$ const or $c_{a}^{2}=0$. For both quintessential scalar fields the potential $U(Q)$ and time dependence of $Q$ are constructed for the same cosmological model and it is shown that the accelerated expansion of the Universe is caused by the effect of the rolling down of the potential to the minimum (Fig. 2). In the QCDMmodel with $w=$ const the ratio $\rho_{M} / \rho_{Q} \rightarrow \infty$ when $a \rightarrow 0$, while in QCDM-model with $c_{a}^{2}=0 \rho_{M} / \rho_{Q} \rightarrow$ $\left(1-\Omega_{Q}\right) /\left(1+w_{0}\right) \Omega_{Q}$ and $w \rightarrow 0$ when $a \rightarrow 0$. In the early epoch $w$-quintessence is a dynamically unsubstantial like cosmological constant while $c_{a}^{2}$-quintessence mimics dust matter $(w \approx 0)$ at $a \ll 1$ and cosmological constant $(w=-1)$ at $a \gg 1$. The dependence of acceleration parameter on redshift is a bit different for them (Fig. 1) but close to the $\Lambda$ CDM-model and indistinquishable observationally now. 
Asymptotic analysis of the systems of evolutionary equations for gauge-invariant perturbations has shown that adiabatic initial conditions for non-relativistic matter and $w$ - and $c_{a}^{2}$-quintessence are allowed. The numerical integration of these systems give time dependences of gauge-invariant variables for matter and quintessence scalar perturbations (Fig. 3, 4). The main conclusion deduced from them is the following: the magnitudes of the adiabatic matter density perturbations grow similarly in the $\Lambda$ CDM-model, while for quintessence $D_{g}^{(Q)}, D_{s}^{(Q)}$ are constant and $D^{(Q)}, V^{(Q)}$ grow before the particle horizon entry but all variables decay after that in such a way that at the current epoch they are approximately two orders lower than the corresponding quantities for dust matter on supercluster scales. Therefore, on subhorizon scales the quintessential scalar field is smoothed out while the matter is clustered.

The quintessential scalar fields studied here suppress the growth of matter density perturbations and the magnitude of gravitational potential (Fig. 6). In these QCDM-models - unlike the $\Lambda$ CDM ones - such a suppression is scale dependent and more visible for $c_{a^{-}}^{2}$ quintessence. Such features of quintessence are important for calculations of the matter density power spectrum at different redshifts and the power spectrum of CMB temperature fluctuations in the range of scales of the late integrated Sachs-Wolfe effect. That can be used for the interpretation of data of current and planned experiments in order to identify the nature of dark energy.

\section{ACKNOWLEDGMENTS}

This work was supported by the project of Ministry of Education and Science of Ukraine "The linear and non-linear stages of evolution of the cosmological perturbations in models of the multicomponent Universe with dark energy" (state registration number 0107U002062) and the research program of National Academy of Sciences of Ukraine "The exploration of the structure and components of the Universe, hidden mass and dark energy (Cosmomicrophysics)" (state registration number 0107U007279). The authors are thankful also to $\mathrm{Yu}$. Kulinich for useful discussions.
[1] S. Apunevych, B. Venhlyovska, Yu. Kulinich, B. Novosyadlyj, Kinematics Phys. Celest. Bodies 23, 45 (2007); arXiv: 0802.0599 [astro-ph].

[2] J. M. Bardeen, Phys. Rev. D 22, 1882 (1980).

[3] N. Bartolo, P.-S. Corasaniti, A. R. Liddle, M. Malquarti, Phys. Rev. D 70, 043532 (2004).

[4] R. Bean, O. Dore, Phys. Rev. D 69, 083503 (2004).

[5] C. L. Bennett et al., Astrophys. J. Suppl. 148, 1 (2003).

[6] S. Bludman, in VI Reunion Anual Sociedad Chilena de Astronomia (SOCHIAS) (Valparaiso, Chile, 2007), p. 9.

[7] R. Bousso, Gen. Relativ. Gravit. 40, 607 (2008).

[8] S. M. Carroll, Living Rev. Rel. 4, 1 (2001).

[9] S. M. Carroll, W. H. Press, E. L. Turner, Ann. Rev. Astron. Astrophys. 30, 499 (1992).

[10] M. Chevallier, D. Polarski, Int. J. Mod. Phys. D 10, 213 (2001).

[11] E. J. Copeland, M. Sami, S. Tsujikawa, Int. J. Mod. Phys. D 15, 1753 (2006).

[12] R. Dave, R. R. Caldwell, P. J. Steinhardt, Phys. Rev. D 66, 023516 (2002).

[13] S. DeDeo, R. R. Caldwell, P. J. Steinhardt, Phys. Rev. D 67, 103509 (2003).

[14] M. Doran, C. M. Muller, G. Schafer, C. Wetterich, Phys. Rev. D 68, 063505 (2003).

[15] R. Durrer, J. Phys. Stud. 5, 177 (2001).

[16] R. Durrer, R. Maartens, Gen. Relativ. Gravit. 40, 301 (2008).

[17] S. Dutta, I. Maor, Phys. Rev. D 75, 063507 (2007).

[18] J. K. Erickson, R. R. Caldwell, P. J. Steinhardt, C. Armendariz-Picon, V. Mukhanov, Phys. Rev. Lett. 88, 121301 (2007).

[19] J. A. Frieman, M. S. Turner, D. Huterer, Ann. Rev. Astron. Astrophys. 46, 385, (2008).

[20] R. Hlozek, V. Cortes, C. Clarkson, B. Bassett, Gen. Relativ. Gravit. 40, 285 (2008).
[21] W. Hu, Astrophys. J. 506, 485 (1998).

[22] W. Hu, D. Eisenstein, Phys. Rev. D 59, 083509 (1999).

[23] H. Kodama, M. Sasaki, Prog. Theor. Phys. Suppl. 78, 1 (1984).

[24] E. Komatsu et al., Astrophys. J. Suppl. Ser. 180, 330 (2007).

[25] Yu. Kulinich, B. Novosyadlyj, V. Pelykh, J. Phys. Stud. 180, 473, (2007).

[26] M. Kunz, astro-ph/0702615 (2007).

[27] E. V. Linder, Phys. Rev. D 76, 063010 (2006).

[28] E. V. Linder, Gen. Relativ. Gravit. 40, 329 (2008)

[29] J. Liu, Phys. Rev. D 69, 083504 (2004).

[30] D. F. Mota, D. J. Shaw, J. Silk, Astrophys. J. 675, 29 (2008).

[31] C.-P. Ma, E. Bertschinger, Astrophys. J. 455, 7 (1995).

[32] B. Novosyadlyj, J. Phys. Stud. 11, 226 (2007).

[33] T. Padmanabhan, Phys. Rep. 380, 235 (2003).

[34] P. J. E. Peebles, Principles of physical cosmology (Princeton University Press, Princeton, 2001), p. 718.

[35] P. J. E. Peebles, B. Ratra, Rev. Mod. Phys. 75, 559 (2003).

[36] V. Sahni, T. D. Saini, A. A. Starobinsky, U. Alam, J. Exp. Theor. Phys. Lett. 77, 201 (2003).

[37] V. Sahni, A. A. Starobinsky, Int. J. Mod. Phys. D 9, 373 (2000).

[38] V. Sahni, A. A. Starobinsky, Int. J. Mod. Phys. D 15, 2105 (2006).

[39] U. Seljak, M. Zaldarriaga, Astrophys. J. 469, 437 (1996).

[40] O. Sergijenko, B. Novosyadlyj, Kinematics Phys. Celest. Bodies 24, 345 (2008); arXiv: 0805.3782 [astro-ph] (2008).

[41] O. Sergijenko, Yu. Kulinich, B. Novosyadlyj, V. Pelykh, Kinematics Phys. Celest. Bodies 25, 259 (2009), arXiv: 0809.3349 [astro-ph] (2008).

[42] I. L. Shapiro, J. Sola, arXiv: 0808.0315 [astro-ph] (2008). 
[43] D. N. Spergel et al., Astrophys. J. Suppl. Ser. 170, 377 (2007).

[44] S. Unnikrishnan, H. K. Jassal, T. R. Seshadri, Phys. Rev. D 78, 123504 (2008).

[45] J. Weller, A. M. Lewis, Mon. Not. R. Astron. Soc. 346,
987 (2003).

[46] M. Zaldarriaga, U. Seljak, Astrophys. J. Suppl. Ser. 29, 431 (1999).

[47] V. I. Zhdanov, G. Ivashchenko, Kinematics Phys. Celest. Bodies 25, 73 (2009).

\title{
ЕВОЛЮЦІЯ СКАЛЯРНИХ ЗБУРЕНЬ У КОСМОЛОГІЇ З КВІНТЕСЕНЦІЙНОЮ TEMНОЮ ЕНЕРГІЕЮ
}

\author{
Б. Новосядлий, О. Сергієнко \\ Астрономічна обсерваторія Львівсъкого начіонального університету імені Івана Франка, \\ вул. Кирила і Мефодія, 8, 79005, Лъвів
}

\begin{abstract}
Досліджено динаміку розширення Всесвіту та еволюцію скалярних збурень у моделях із квінтесенційними скалярними полями $Q$ (класичний лагранжіан $L=\frac{1}{2} Q_{; i} Q^{; i}-U(Q)$ ), що задовольняють додаткову умову сталості параметра рівняння стану $(w=$ const), або нульового значення адіабатичної швидкості звуку $\left(c_{a}^{2}=0\right)$. Обидва квінтесенційні поля проаналізовано в межах одної космологічної моделі. Показано, що прискорене розширення Всесвіту зумовлене ефектом "скочування" поля до мінімуму потенціалу. У ранню епоху вплив квінтесенції з $w=$ const на динаміку розширення Всесвіту нехтівно малий (подібно, як у випадку космологічної сталої), тоді як поле з $c_{a}^{2}=0$ веде себе як пилоподібна матерія. У далекому майбутньому скалярне поле із $c_{a}^{2}=0$ за своїми проявами буде подібним на космологічну сталу.

Отримано аналітичні розв'язки системи рівнянь, що описують еволюцію калібрувально-інваріантних збурень метрики простору-часу, густини і швидкості матерії та квінтесенції, для ранньої стадії еволюції Всесвіту та числові для всієї історії аж до сучасної епохи включно. Показано, що амплітуди адіабатичних збурень матерії зростають однаково в обох моделях (і подібно до зростання в $\Lambda$ CDM-моделі), але часові залежності різних амплітуд збурень квінтесенції різні: калібрувально-інваріантні змінні $D_{g}^{(Q)}$ і $D_{s}^{(Q)}$ загасають від початкової сталої величини після входження збурення в горизонт частинки, тоді як $D^{(Q)}$ i $V^{(Q)}$ зростають на ранній стадії до входження в горизонт і загасають після входження, коли квінтесенція починає домінувати за густиною, а гравітаційний потенціал загасати. У сучасну епоху вони приблизно на два порядки менші за відповідні амплітуди збурень матерії. Отже, на масштабах, менших за горизонт частинки, квінтесенційні скалярні поля є майже однорідними, тоді як матерія кластеризованою.

Показано також, що обидва квінтесенційні скалярні поля сповільнюють зростання збурень густини матерії та гравітаційного потенціалу. У цих QCDM-моделях, на відміну від $\Lambda$ CDM-моделей, таке сповільнення залежить від масштабу і помітніше для квінтесенції з $c_{a}^{2}=0$.
\end{abstract}

